\title{
Bispectrum analysis of the unresolved gamma-ray background
}

\author{
Ebo Peerbooms ${ }^{a, *}$ \\ ${ }^{a}$ University of Amsterdam, GRAPPA Institute \\ 1098 XH Amsterdam, The Netherlands \\ ${ }^{b}$ University of Amsterdam, Institute for Theoretical Physics \\ 1098 XH Amsterdam, The Netherlands \\ E-mail: e.peerbooms@uva.nl
}

In recent years, properties of the unresolved gamma ray background (UGRB) have been constrained by measuring the anisotropy in the form of the angular power spectrum (APS). The energy dependence of the anisotropy has been found to be consistent with a broken power law, suggesting the existence of two distinct source classes above and below $\sim 2 \mathrm{GeV}$. In this work, we aim to go beyond the angular power spectrum, and use the bispectrum to further constrain the source classes that contribute to the UGRB. As in the case of the APS, for a skymap consisting of unresolved, randomly distributed point sources, we expect the bispectrum to be independent of multipole and therefore to be fully characterised by a single amplitude bsrc. We adapt the formalism developed in the context of CMB research and apply the resulting analysis pipeline to Fermi-LAT data in the energy range $0.7 \mathrm{GeV}-1 \mathrm{TeV}$. We verify the robustness of our analysis pipeline by applying it to simulated realizations with a predetermined value of the bispectrum amplitude. Additionally, bispectrum amplitudes obtained from the UGRB data are compared to simulated, purely isotropic realizations of the UGRB in order to test for deviations from pure Poisson noise across the entire energy range. Finally, we check if the energy-dependence of the bispectrum amplitude is consistent with the same broken power-law is in the case of the APS.

$37^{\text {th }}$ International Cosmic Ray Conference (ICRC 2021)

July 12 th - 23rd, 2021

Online - Berlin, Germany

\footnotetext{
${ }^{*}$ Presenter
} 


\section{Introduction}

The unresolved gamma-ray background (UGRB) is the component of the gamma-ray sky that remains after emission from the Milky Way and known gamma-ray sources has been subtracted. The precise origin of the UGRB and the nature of source populations contributing is still an open question [1]. Believed to be mostly extragalactic in origin [2], the UGRB consists of sources that are too faint to be resolved individually. Through the analysis of data obtained by the Fermi Large Area Telescope (Fermi-LAT) [3], significant progress has been made in our understanding of the nature of the UGRB [4]. Source classes known to contribute to the UGRB include blazars, misaligned AGNs, star-forming galaxies and millisecond pulsars [1,5-7]. Other components may include galaxy clusters, type Ia supernovae and isotropic processes [1]. Out of these sources, blazars are believed to constitute the dominant source class [5, 8]. Recent studies constrain the contribution from blazars to the total intensity to be $\lesssim 20 \%$, while simultaneously contributing most of the measured anisotropy [5]. In this work, the pipeline for the analysis of the UGRB through the angular power spectrum [9] has been extended to the bispectrum.

\section{Angular power spectrum}

\subsection{Introduction}

The angular power spectrum (APS) is defined as the spherical harmonic transform of the twopoint correlation function. A spherical harmonic transform is analogous to the Fourier transform, but uses a basis of spherical harmonics. In the study of the UGRB, the intensity map is decomposed into spherical harmonics as follows

$$
a_{l m}=\int_{4 \pi} d \Omega I(\Omega) Y_{l m}^{*}(\Omega)
$$

The $a_{l m}$ are known as the harmonic coefficients and quantify the contribution from features at a given angular scale. Each angular scale is characterized by a label $l$, which are known as multipoles. The angular scale in degrees is related to a specific multipole $l$ by the relation $\theta_{l}=180^{\circ} / l$. The intensity map can be recovered from the harmonic coefficients by the inverse harmonic transform [10], given by

$$
I(\Omega)=\sum_{l m} a_{l m} Y_{l m}^{*}(\Omega) .
$$

The APS measures the amount of angular multiple at each multipole, it is given by

$$
C_{l}=\frac{1}{2 l+1} \sum_{m=-l}^{m=l}\left|a_{l m}\right|^{2} .
$$

It is essentially the average over the norm of the $a_{l m}$ for a given $l$, since for each $l$ there are $2 l+1$ orthogonal harmonic functions.

\subsection{Measuring the APS}

In the context of the UGRB, the APS is used to quantify the contribution from unresolved point sources to the measured data $[5,11,12]$. For an isotropic distribution of point sources, the 
APS is essentially flat, since there is clustering of sources at any preferred angular scale [11]. By measuring the amplitude of this flat signal, which is denoted by $C_{P}$, the properties and abundance of the underlying source population can be inferred. Having measured $C_{P}$ due to unresolved sources, the contribution from a specific source population can be constrained through its source-count distribution $d N / d S$ [8] by integrating this distribution up to the source detection threshold flux $S_{t}$

$$
C_{P}^{\text {source }}=\int_{0}^{S_{t}} d S \frac{d N}{d S} S^{2}
$$

By comparing the expected $C_{P}^{\text {source }}$ obtained this way to the measured $C_{P}$, upper limits can be placed on the contribution from a specific source to the total angular power.

Due to the finite exposure time of the instrument, the APS comes with an additional contribution due to photon noise. By measuring the events, one is essentially taking a Poisson sample of the underlying flux distribution. Therefore the measured events $n(\Omega)$ at a given location on the sky is given by $n(\Omega)=\bar{n}(\Omega)+\delta n$. The discrepancy $\delta n$ from the expected number of events $\bar{n}$ is the source of this additional noise.

In addition to this noise, the finite angular resolution of the instrument has the effect of smearing the measured events into pixels adjacent to the true location of an emitting source. This leads to suppression of power at small angular scales, since the finite angular resolution imposes a cut-off scale to the maximum multipole. This effect is quantified in a beam window function $w_{l}$ [12].

The main source of high energy gamma-rays in the sky is galactic diffuse emission (GDE) originating from the Milky Way Galaxy [13]. In order to study the emission from the UGRB, regions of the sky where the GDE is the dominant source of emission need to be masked. This masking introduces an additional bias to the measured APS. The fraction of the sky remaining after masking is denoted by $f_{\text {sky }}$.

Taking into account all these effects, the measured APS is given by

$$
C_{l}^{\text {data }}=\left(C_{l}^{\text {signal }} w_{l}^{2}+C_{N}\right) f_{\text {sky }} .
$$

Inverting this relation results yields

$$
C_{l}^{\mathrm{signal}}=\frac{C_{l}^{\mathrm{data}} / f_{\mathrm{sky}}-C_{N}}{w_{l}^{2}} .
$$

From this point on, we use just $C_{l}$ when referring to $C_{l}^{\text {signal }}$. The contribution from unresolved point sources is obtained by fitting the signal APS to a constant value. This is done through the minimization of

$$
\chi^{2}=\sum_{l} \frac{\left(C_{l}-C_{P}\right)^{2}}{\sigma_{l}^{2}}
$$

with the errors on the $C_{l}$ given by [14]

$$
\sigma_{l}^{2}=\frac{2}{(2 l+1) f_{\mathrm{sky}}}\left(C_{l}+\frac{C_{N}}{w_{l}^{2}}\right)^{2}
$$




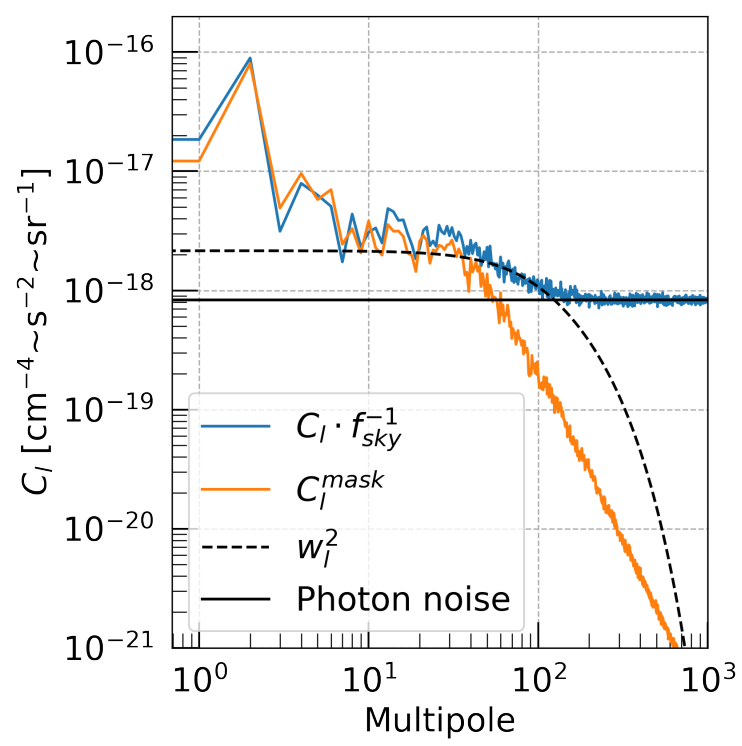

Figure 1: Raw angular power spectrum obtained using HEALPIX in the energy bin $0.86-1.02 \mathrm{GeV}$ (blue). Also shown is the angular power of the mask normalized to the data power spectrum (orange). The dotted line corresponds to the beam window function for this energy bin and the black line is the estimate of the photon noise. At low multipoles, the raw power spectrum is dominated by masking effects.

The sum in eq. (7) in principle runs over all multipoles. However, as mentioned, masking and window functions impart a bias to the signal at low and high multipoles respectively. Therefore, most authors choose to limit this analysis to a specific range of multipoles that is dependent on the properties of the mask and the window functions for the energy range under consideration $[5,9,12]$.

\section{Angular bispectrum}

\subsection{Introduction}

The angular bispectrum is the harmonic transform of the three-point correlation function [15]. The full bispectrum is given by

$$
B_{l_{1} l_{2} l_{3}}^{m_{1} m_{2} m_{3}} \equiv\left\langle a_{l_{1} m_{1}} a_{l_{2} m_{2}} a_{l_{3} m_{3}}\right\rangle
$$

Due to statistical isotropy of the universe, the angle-averaged bispectrum

$$
B_{l_{1} l_{2} l_{3}}=\sum_{m}\left(\begin{array}{ccc}
l_{1} & l_{2} & l_{3} \\
m_{1} & m_{2} & m_{3}
\end{array}\right) B_{l_{1} l_{2} l_{3}}^{m_{1} m_{2} m_{3}}
$$

can be used without loss of physical information $[15,16]$. The matrix appearing in the equation above is the Wigner- $3 j$ symbol which ensures that $l_{1}, l_{2}$ and $l_{3}$ form a triangle in harmonic space. All physical information is encoded in a quantity known as the reduced bispectrum, which is defined in the following way

$$
b_{l_{1} l_{2} l_{3}}=\left(\begin{array}{ccc}
l_{1} & l_{2} & l_{3} \\
0 & 0 & 0
\end{array}\right)^{-1} \sqrt{\frac{4 \pi}{\left(2 l_{1}+1\right)\left(2 l_{2}+1\right)\left(2 l_{3}+1\right)}} B_{l_{1} l_{2} l_{3}}
$$


As with the APS, the reduced bispectrum due to unresolved point sources is independent of multipole [17] and thus characterized by a single amplitude $b_{P}$ (usually referred to a $b_{\text {src }}$ in the literature [18]).

\subsection{Measuring the reduced bispectrum}

Since a flat reduced bispectrum is expected from a population of unresolved point sources, it should in principle be sufficient to measure only the diagonal components of the reduced bispectrum, i.e. those for which $l_{1}=l_{2}=l_{3}$. A computationally effective way to calculate the reduced bispectrum is given by $[16,19]$

$$
b_{l_{1} l_{2} l_{3}}=\frac{1}{4 \pi}\left(\begin{array}{ccc}
l_{1} & l_{2} & l_{3} \\
0 & 0 & 0
\end{array}\right)^{-2} \sqrt{\frac{4 \pi}{\left(2 l_{1}+1\right)\left(2 l_{2}+1\right)\left(2 l_{3}+1\right)}} \int d \Omega e_{l_{1}}(\Omega) e_{l_{2}}(\Omega) e_{l_{3}}(\Omega)
$$

For all $l$ equal, this can be written as follows

$$
b_{l} \equiv b_{l l l}=\frac{2}{\pi} \frac{\Gamma\left(\frac{1-l}{2}\right)^{2}}{\Gamma\left(\frac{1+l}{2}\right)} \frac{\Gamma\left(\frac{2+l}{2}\right)^{3} \Gamma\left(\frac{3(1+l)}{2}\right)}{\Gamma\left(1+\frac{3 l}{2}\right)} \int d \Omega\left[\frac{q_{l}(\Omega)}{2 l+1}\right]^{3}
$$

where $q_{l}$ is the partial harmonic transform for a given $l$ [20]

$$
q_{l}=\sum_{m} a_{l m} Y_{l m}(\Omega)
$$

and $\Gamma$ is the gamma function. As with the APS, the measured $b_{l}$ need to be corrected for the instrument response, a contribution from photon noise and the effect of masking. The reduced bispectrum due to the signal is given by

$$
b_{l}^{\text {signal }}=\frac{b_{l}^{\text {data }} / f_{\text {sky }}-b_{N}}{w_{l}^{3}}
$$

completely analogous to the APS analysis. Again, the contribution from point sources is measured by appropriate modification of eq. (7) for which the error in this case is given by [16]

$$
\sigma_{l}^{2}=\left\langle C_{l}+C_{N} w_{l}^{-2}\right\rangle^{3}\left(\begin{array}{ccc}
l_{1} & l_{2} & l_{3} \\
0 & 0 & 0
\end{array}\right)^{-2} \frac{24 \pi}{(2 l+1)^{3}}
$$

\section{Results}

The diagonal bispectrum coefficients were measured in 10 logarithmically spaced energy bin in the range between $700 \mathrm{MeV}$ and $1 \mathrm{TeV}$ using the Pass 8 Fermi-LAT data [3, 21]. The range of multipoles was restricted to the range $l \in[2,1000]$. For the fitting of the amplitude due to unresolved point sources to the data, this range was further restricted to the range $l \in\left[100, l_{\max }\right]$, where $l_{\max }$ is the minimum multipole for which the suppression by the window function is smaller than a factor of 10 . The choice for the lower limit was motivated due to residual effects from 

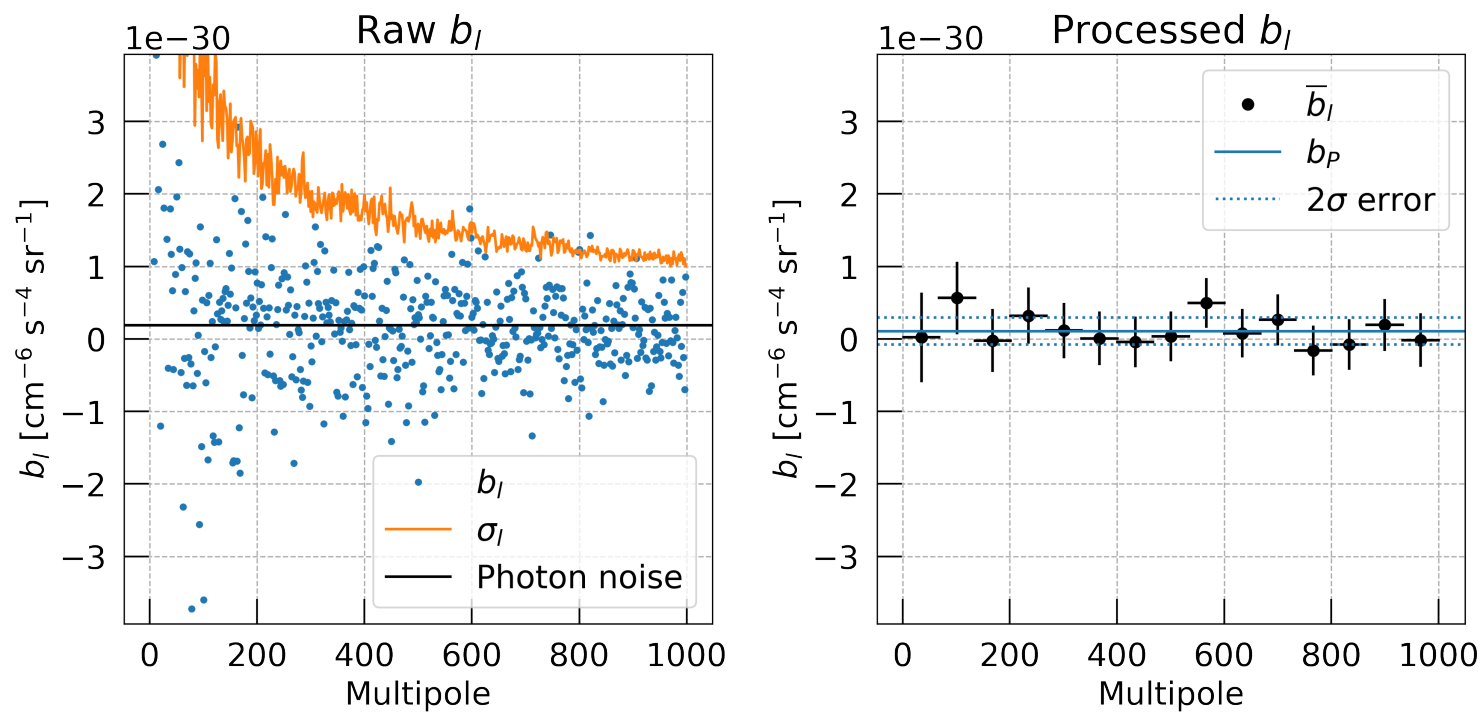

Figure 2: Left: Diagonal bispectrum coefficients measured in $26.5-54.7 \mathrm{GeV}$ energy bin (blue dots). Also shown is the error estimate as given by eq. (16) (orange line) and the photon noise offset estimate. Right: Binned coefficients after correction as in eq. (15) and obtained value of $b_{P}$ and 2-sigma uncertainty.
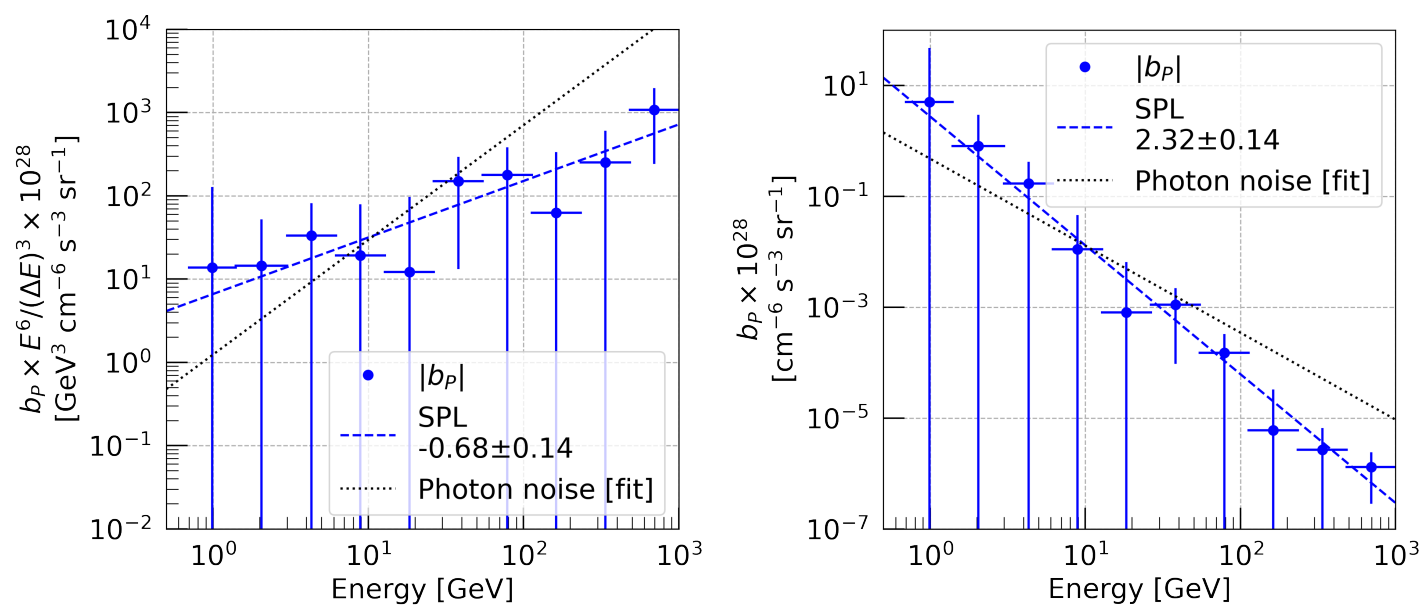

Figure 3: Power law fit to the reduced bispectrum energy spectrum. Left: absolute value of the measured reduced bispectrum normalized by $E^{6} /(\Delta E)^{3}$. Right: absolute value of the measured reduced bispectrum. Note that both fits are consistent with each other, $-0.68+3=2.32$, since we need to add 3 to the power law index of the normalized fit to convert back to values in terms of intensity spectra [9]. Large downward error bars indicate that the measurements are consistent with zero.

the application of a mask, which shows up in both the APS and the bispectrum as an increasing amplitude at low multipoles. For each energy range, the bispectrum coefficients were binned into 15 linearly spaced multipole bins, assigning the best-fit $b_{l}$ to each bin by minimizing a chi-squared function for the multipoles in that bin. Absolute values of the obtained normalized (by $E^{6} / \Delta E^{3}$ ) 
bispectrum amplitudes were fitted to a power law,

$$
N \times\left(\frac{E}{\mathrm{GeV}}\right)^{-\alpha}
$$

for which we find an index $\alpha=2.32 \pm 0.14$. The absolute value was taken to stabilize the fitting procedure and because the relative sign of the measured bispectrum is not of interest in the context of this analysis.

\section{Discussion and conclusion}

We have adapted the analysis pipeline for the study of the angular power spectrum to the bispectrum. Using diagonal coefficients allows for a correspondence between both methods. The obtained energy dependence of the bispectrum amplitudes follows a power law with an index of $2.32 \pm 0.14$. For blazars, it has been shown that the energy dependence of $C_{P}$ obeys a power law with an index in the range 1.8-2.6 [9]. Such a population would result in a power law energy dependence for $b_{P}$ in the range $\sim 3-4$. Also note that for each energy bins the measured bispectrum amplitudes are consistent with zero. We thus conclude that the pipeline seems insufficiently sensitive to the small signal contribution to the reduced bispectrum.

\section{References}

[1] M. Fornasa and M. A. Sánchez-Conde, The nature of the diffuse gamma-ray background, Physics Reports 598 (Oct, 2015) 1-58.

[2] M. Ackermann, M. Ajello, A. Albert, W. B. Atwood, L. Baldini, J. Ballet et al., The spectrum of isotropic diffuse gamma-ray emission between 100 mev and $820 \mathrm{gev}$, The Astrophysical Journal 799 (Jan, 2015) 86.

[3] S. Abdollahi, F. Acero, M. Ackermann, M. Ajello, W. B. Atwood, M. Axelsson et al., Fermi large area telescope fourth source catalog, The Astrophysical Journal Supplement Series 247 (Mar, 2020) 33.

[4] L. Collaboration, Fermi large area telescope third source catalog, 2015.

[5] A. Cuoco, E. Komatsu and J. M. Siegal-Gaskins, Joint anisotropy and source count constraints on the contribution of blazars to the diffuse gamma-ray background, Physical Review D - Particles, Fields, Gravitation and Cosmology 86 (2012) , [1202 . 5309].

[6] C. D. Dermer, The extragalactic $\gamma$ ray background, AIP Conference Proceedings (2007).

[7] D. Bhattacharya, P. Sreekumar and R. Mukherjee, Contribution from unresolved discrete sources to the extragalactic gamma-ray background (egrb), Research in Astronomy and Astrophysics 9 (Oct, 2009) 1205-1214.

[8] L. Marcotulli, M. Di Mauro and M. Ajello, Source-count distribution of gamma-ray blazars, The Astrophysical Journal 896 (Jun, 2020) 6. 
[9] M. Ackermann, M. Ajello, L. Baldini, J. Ballet, G. Barbiellini, D. Bastieri et al., Unresolved gamma-ray sky through its angular power spectrum, Physical Review Letters 121 (Dec, 2018) .

[10] G. Rocha, M. P. Hobson, S. Smith, P. Ferreira and A. Challinor, Simulation of non-Gaussian cosmic microwave background maps, Monthly Notices of the Royal Astronomical Society 357 $(02,2005) 1-11$, [https://academic . oup.com/mnras/article-pdf/357/1/1/3464231/357-1-1.pdf].

[11] M. Ackermann, M. Ajello, A. Albert, L. Baldini, J. Ballet, G. Barbiellini et al., Anisotropies in the diffuse gamma-ray background measured by the fermi lat, Physical Review D 85 (Apr, 2012).

[12] M. Fornasa, A. Cuoco, J. Zavala, J. M. Gaskins, M. A. Sánchez-Conde, G. Gomez-Vargas et al., Angular power spectrum of the diffuse gamma-ray emission as measured by the fermi large area telescope and constraints on its dark matter interpretation, Physical Review D 94 (Dec, 2016) .

[13] D. Malyshev and D. W. Hogg, Statistics of gamma-ray point sources below the fermi detection limit, The Astrophysical Journal 738 (Aug, 2011) 181.

[14] L. Knox, Determination of inflationary observables by cosmic microwave background anisotropy experiments, Physical Review D 52 (Oct, 1995) 4307-4318.

[15] M. G. Santos, A. Heavens, A. Balbi, J. Borrill, P. G. Ferreira, S. Hanany et al., Multiple methods for estimating the bispectrum of the cosmic microwave background with application to the maxima data, Monthly Notices of the Royal Astronomical Society 341 (May, 2003) 623-643.

[16] E. Komatsu, B. D. Wandelt, D. N. Spergel, A. J. Banday and K. M. Gorski, Measurement of the cosmic microwave background bispectrum on the cobe dmr sky maps, The Astrophysical Journal 566 (2002) 19-29, [0107605].

[17] E. Komatsu, Hunting for primordial non-gaussianity in the cosmic microwave background, Classical and Quantum Gravity 27 (May, 2010) 124010.

[18] E. Komatsu, J. Dunkley, M. R. Nolta, C. L. Bennett, B. Gold, G. Hinshaw et al., Five-year wilkinson microwave anisotropy probe observations: Cosmological interpretation, The Astrophysical Journal Supplement Series 180 (Feb, 2009) 330-376.

[19] E. Komatsu and D. N. Spergel, Acoustic signatures in the primary microwave background bispectrum, Physical Review D 63 (Feb, 2001) .

[20] D. N. Spergel and D. M. Goldberg, Microwave background bispectrum. i. basic formalism, Physical Review D 59 (Apr, 1999) .

[21] P. Bruel, T. H. Burnett, S. W. Digel, G. Johannesson, N. Omodei and M. Wood, Fermi-lat improved pass 8 event selection, 2018. 